\title{
A Threshold-based Linear Parallel Interference Canceller on Fading Channels
}

\author{
V. Tikiya and A. Chockalingam \\ Department of ECE, Indian Institute of Science, Bangalore 560012, India
}

\begin{abstract}
In this paper, we obtain closed-form expressions for the bit error rate (BER) of conventional linear parallel interference canceller (LPIC), where the interference from all other users are estimated and cancelled on Rayleigh fading and diversity channels. We also propose an improved LPIC scheme which cancels the interference from only those users whose instantaneous SNRs exceed a certain threshold on Rayleigh fading channels. We obtain the optimum threshold by optimizing an approximate expression for the SIR at the canceller output. We show that the proposed threshold-based LPIC scheme performs better than the matched filter (MF) detector as well as the conventional LPIC scheme.
\end{abstract}

Keywords - Parallel interference cancellation, linear PIC, fading channels.

\section{INTRODUCTION}

Parallel interference cancellation (PIC) is a multiuser detection scheme where a desired user's decision statistic is obtained by subtracting an estimate of the multiple access interference (MAI) from the received signal [1],[2]. PIC lends itself to a multistage implementation where the decision statistics of the users from the previous stage are used to estimate and cancel the MAI in the current stage, and a final decision statistic is obtained at the last stage of the PIC. When an estimate of the MAI is obtained from the hard bit decisions from the previous stage, it is called non-linear PIC, which is also termed as 'Hard-decision PIC' (HPIC). The multistage PIC scheme originally proposed by Varanasi and Aazhang in [3] and several other schemes considered in the literature (e.g., [4]) are of this type. On the other hand, MAI estimates can be obtained using the soft values of the decision statistics from the previous stage, in which case the PIC is termed as linear PIC (LPIC) [5],[6]. A comparison between the performance of HPIC and LPIC is presented in [5], where, based on a comparison between the mean-square error (MSE) of the MAI estimators, it has been shown that HPIC provides a superior MAI estimator than LPIC. However, there is significant interest in LPIC due to its simplicity in implementation, analytical tractability, and good performance under certain conditions [5]-[7].

It has been also shown that when the accuracy of the MAI estimates is poor (e.g., due to poor channel/SIR conditions), the performance of PIC can become worse than the matched filter (MF) detector where MAI cancellation is not performed [5]. In [7], a multistage weighted cancellation scheme has been proposed for both linear as well as non-linear PIC. In [8], the

This work was supported in part by the Swarnajayanti Fellowship, Department of Science and Technology, New Delhi, Government of India, under Project Ref: No.6/3/2002-S.F. performance of the non-linear PIC scheme in [7] has been analyzed on fading channels using Gaussian approximation of the interference. In this paper, we are concerned with linear PIC schemes on fading channels.

Our contributions in this paper are two-fold; first we obtain closed-form expressions for the bit error rate (BER) of conventional linear PIC, where the interference from all other users are estimated and cancelled on flat Rayleigh fading as well as diversity channels, and second we propose an improved linear PIC scheme which cancels the interference from only those users whose instantaneous SNRs exceed a certain threshold on Rayleigh fading channels. We obtain the optimum threshold by optimizing an approximate expression for the SIR at the canceller output. We show that the proposed threshold-based LPIC scheme performs better than the MF detector as well as the conventional LPIC scheme.

The rest of the paper is organized as follows. Section II presents the system model. Section III presents the BER analysis for the conventional LPIC scheme on fading and diversity channels. Section IV presents the proposed thresholdbased LPIC scheme and the optimum thresholds. Section V presents the performance results and discussions. Conclusions are given in Section VI.

\section{SySTEM MODEL}

Consider a $K$-user synchronous CDMA system where the received signal is given by

$$
y(t)=\sum_{k=1}^{K} A_{k} h_{k} b_{k} s_{k}(t)+n(t), \quad t \in[0, T],
$$

where $b_{k} \in\{+1,-1\}$ is the bit transmitted by the $k$ th user, $T$ is one bit duration, $A_{k}$ is the transmit amplitude of the $k$ th user's signal, $h_{k}$ is the complex channel fade coefficient corresponding to the $k$ th user, $s_{k}(t)$ is the unit energy spreading waveform of the $k$ th user defined in the interval $[0, T]$, i.e., $\int_{0}^{T} s_{k}^{2}(t) d t=1$, and $n(t)$ is the white Gaussian noise with zero mean and variance $\sigma^{2}$. The fade coefficients $h_{k}$ 's are assumed to be i.i.d complex Gaussian r.v's (i.e., fade amplitudes are Rayleigh distributed) with zero mean and $E\left[h_{I}^{2}\right]=$ $E\left[h_{Q}^{2}\right]=1$, where $h_{k I}$ and $h_{k Q}$ are the real and imaginary parts of $h_{k}$. The channel fade is assumed to remain constant over one bit interval.

We consider a two-stage LPIC at the receiver. The first stage is a conventional MF detector, which is a bank of $K$ correlators, each matched to a different user's spreading waveform. 
The received vector $\mathbf{y}^{(1)}$ at the output of the MF stage (the superscript (1) in $\mathbf{y}^{(1)}$ denotes the first stage) is given by

$$
\mathbf{y}^{(1)}=\left[y_{1}^{(1)}, y_{2}^{(1)}, \cdots, y_{K}^{(1)}\right],
$$

where $y_{k}^{(1)}$ is the $k$ th user MF output, given by

$$
y_{k}^{(1)}=A_{k} h_{k} b_{k}+\sum_{j=1, j \neq k}^{K} \rho_{j k} A_{j} h_{j} b_{j}+n_{k},
$$

where $\rho_{j k}$ is the cross-correlation between the $j$ th and $k$ th users' spreading waveforms, given by $\rho_{j k}=\int_{0}^{T} s_{j}(t) s_{k}(t) d t$, and $n_{k}$ 's are complex Gaussian with zero mean and $E\left[n_{j} n_{k}^{*}\right]=$ $2 \sigma^{2}$ when $j=k$ and $E\left[n_{j} n_{k}^{*}\right]=2 \sigma^{2} \rho_{j k}$ when $j \neq k$. The received vector $\mathbf{y}^{(1)}$ (without hard decision) is used for MAI estimation and cancellation in the second stage.

In the second stage, we consider two different cancellation schemes. The first scheme is the conventional LPIC, where the interference from all the users is estimated and cancelled. The second scheme is our proposed threshold-based LPIC, where only those users whose instantaneous SNRs exceed a certain threshold are cancelled.

\section{BER OF CONVENTIONAL LPIC}

In the conventional LPIC, the interference from all other users are estimated and cancelled, i.e., the MAI estimate for the $k$ th user is obtained by multiplying $y_{j}^{(1)}$ with $\rho_{j k}$ for all $j \neq k$ and summing them up, i.e., $\sum_{j \neq k} \rho_{j k} y_{j}^{(1)}$ is the MAI estimate for user $k$. Accordingly, the bit decision for the $k$ th user after interference cancellation in the second stage is given by

$$
\hat{b}_{k}^{(2)}=\operatorname{sgn}\left(\operatorname{Re}\left(h_{k}^{*}\left(y_{k}^{(1)}-\sum_{j=1, j \neq k}^{K} \rho_{j k} y_{j}^{(1)}\right)\right)\right)
$$

The interference cancelled output of the second stage for the $k$ th user, $y_{k}^{(2)}$, can be written as

$$
\begin{aligned}
y_{k}^{(2)} & =y_{k}^{(1)}-\sum_{j=1, j \neq k}^{K} \rho_{j k} y_{j}^{(1)} \\
& =A_{k} h_{k} b_{k}\left(1-\sum_{j=1, j \neq k}^{K} \rho_{j k}^{2}\right)-I_{2}+N_{2},
\end{aligned}
$$

where

$$
\begin{gathered}
I_{2}=\sum_{j=1, j \neq k}^{K} \rho_{j k} \sum_{\substack{i=1 \\
i \neq j, k}}^{K} \rho_{i j} A_{i} h_{i} b_{i}, \\
N_{2}=n_{k}-\sum_{j=1, j \neq k}^{K} \rho_{j k} n_{j} .
\end{gathered}
$$

The terms $I_{2}$ and $N_{2}$ in (5) represent the interference and noise terms introduced due to the imperfect cancellation that results in using the soft output values from the MF stage for MAI estimation. It is noted that both $N_{2}$ and $I_{2}$ are linear combinations of Gaussian r.v's with zero mean. The variance of $I_{2}, \sigma_{I_{2}}^{2}$, is given by

$$
\sigma_{I_{2}}^{2}=\sum_{\substack{i=1 \\ i \neq k}}^{K} \sum_{\substack{j=1 \\ j \neq i, k}}^{K} \sum_{\substack{l=1 \\ l \neq i, k}}^{K} A_{i}^{2} \rho_{j k} \rho_{l k} \rho_{i j} \rho_{i l}
$$

and the variance of $N_{2}, \sigma_{N_{2}}^{2}$, is given by

$$
\sigma_{N_{2}}^{2}=\sigma^{2}\left(1-\sum_{\substack{j=1 \\ j \neq k}}^{K} \rho_{j k}^{2}+\sum_{\substack{i=1 \\ i \neq k}}^{K} \sum_{\substack{j=1 \\ j \neq k, i}}^{K} \rho_{i k} \rho_{j k} \rho_{i j}\right)
$$

The average SIR for the $k$ th user at the output of the second stage, $\overline{S I R}_{k}^{(2)}$, is then given by

$$
\overline{S I R}_{k}^{(2)}=\frac{A_{k}^{2}\left(1-\sum_{j=1, j \neq k}^{K} \rho_{j k}^{2}\right)^{2}}{\sigma_{I_{2}}^{2}+\sigma_{N_{2}}^{2}} .
$$

The probability of bit error for the $k$ th user at the output of the second stage, $P_{k}^{(2)}$, can be obtained as

$$
P_{k}^{(2)}=\frac{1}{2}\left(1-\sqrt{\frac{\overline{S I R}_{k}^{(2)}}{1+\overline{S I R}_{k}^{(2)}}}\right) .
$$

Suppose we consider diversity with $L$ i.i.d receive antenna paths. The cancellation is done on each path and the resulting outputs are coherently combined. Accordingly, the bit decision for the $k$ th user at the output of the second stage is given by

$$
\hat{b}_{k}^{(2)}=\operatorname{sgn}\left(\operatorname{Re}\left(\sum_{l=1}^{L}\left(h_{k}^{l}\right)^{*} y_{k}^{(2)_{l}}\right)\right),
$$

where $h_{k}^{l}$ denotes the $k$ th user's complex channel coefficient on the $l$ th receive antenna path, and $y_{k}^{(2)_{l}}$ denotes the $k$ th user's interference cancelled output of the second stage on the $l$ th receive antenna path, given by

$$
y_{k}^{(2)_{l}}=y_{k}^{(1)_{l}}-\sum_{j=1, j \neq k}^{K} \rho_{j k} y_{j}^{(1)_{l}} .
$$

On each receive antenna path, the expressions for the variances due to interference, $\sigma_{I_{2}}^{2}$, and due to noise, $\sigma_{N_{2}}^{2}$, remain the same as those for the flat fading case given before. The probability of bit error for the $k$ th user with $L$-path receive diversity can be obtained as [1]

$$
P_{k}^{(2)}=\frac{1}{2}-\frac{1}{2} \sqrt{\frac{\overline{S I R}_{k}^{(2)}}{1+\overline{S I R}_{k}^{(2)}}}\left(1+\sum_{n=1}^{L-1} \frac{1.2 \cdots \cdot(2 n-1)}{n ! 2^{n}\left(1+\overline{S I R}_{k}^{(2)}\right)^{n}}\right),
$$

where $\overline{S I R}_{k}^{(2)}$ is defined as (10). 


\section{THRESHOLD-BASED LPIC}

The conventional LPIC analyzed above performs worse than the MF detector when the reliability of the MAI estimates is poor (as we will see in Sec. V). Here, we propose that by doing selective cancellation, i.e., cancel only those users whose instantaneous received SNRs exceed a certain threshold on fading channels, the performance of the PIC can be improved. This threshold-based cancellation is applicable to both LPIC as well as HPIC. In the following, we consider threshold-based cancellation for LPIC. A key question in this system is how to choose the optimum threshold. We propose two methods for choosing the threshold. For the case of equi-correlated users, we obtain the threshold by optimizing an approximate expression for the SIR at the canceller output. For the case of non-equi-correlated users, we optimize an approximate expression for the mean square error of the MAI estimator.

Consider the equi-correlated case where $\rho_{j k}=\rho, \forall j, k, j \neq$ $k$. For this case, we first derive an expression for the average number of users, $n_{a}$, whose received SNRs exceed a certain threshold, $\gamma$. We then use $n_{a}$ to write an approximate SIR expression which we optimize to obtain the optimum threshold. Let the transmit amplitudes of all the users be the same, i.e., $A_{1}=A_{2}=\cdots A_{K}=A$. Let the instantaneous SNR of the $j$ th user be denoted by $\beta_{j}$, given by $\beta_{j}=\frac{A^{2}\left|h_{j}\right|^{2}}{\sigma^{2}}$. The probability that the first $n$ out of $K-1$ users' (i.e., users other than the desired user $k$ ) received SNRs cross the threshold $\gamma$ is given by

$$
\begin{aligned}
p & =\operatorname{Pr}\left(\beta_{1}>\gamma, \cdots, \beta_{n}>\gamma, \beta_{n+1}<\gamma, \cdots, \beta_{K}<\gamma\right) \\
& =\prod_{\substack{i=1 \\
i \neq k}}^{n} \operatorname{Pr}\left(\beta_{i}>\gamma\right) \cdot \prod_{\substack{i=n+1 \\
i \neq k}}^{K} \operatorname{Pr}\left(\beta_{i}<\gamma\right),
\end{aligned}
$$

since the r.v's $h_{1}, h_{2}, \cdots, h_{k-1}, h_{k+1}, \cdots, h_{K}$ are independent. Also, since $\left|h_{1}\right|^{2}, \cdots,\left|h_{k-1}\right|^{2},\left|h_{k+1}\right|^{2}, \cdots,\left|h_{K}\right|^{2}$ are i.i.d chi-square distributed, the above can be written as

$$
p=\left(e^{-\frac{\gamma \sigma^{2}}{2 A^{2}}}\right)^{n}\left(1-e^{-\frac{\gamma \sigma^{2}}{2 A^{2}}}\right)^{K-1-n} .
$$

Let $b=e^{-\frac{\gamma \sigma^{2}}{2 A^{2}}}$. Considering all possible combinations of $K-1$ users, the probability that $n$ out of $K-1$ users' SNRs cross the threshold $\gamma$ is

$$
p(n ; K-1)=\left(\begin{array}{c}
K-1 \\
n
\end{array}\right) b^{n}(1-b)^{K-1-n} .
$$

The average number of users crossing the threshold is then given by

$$
\begin{aligned}
n_{a} & =\sum_{n=0}^{K-1} n p(n ; K-1) \\
& =(K-1) b .
\end{aligned}
$$

Now considering $n_{a}$ equi-correlated users being cancelled at the second stage, the cancelled output is given by $y_{k}-$

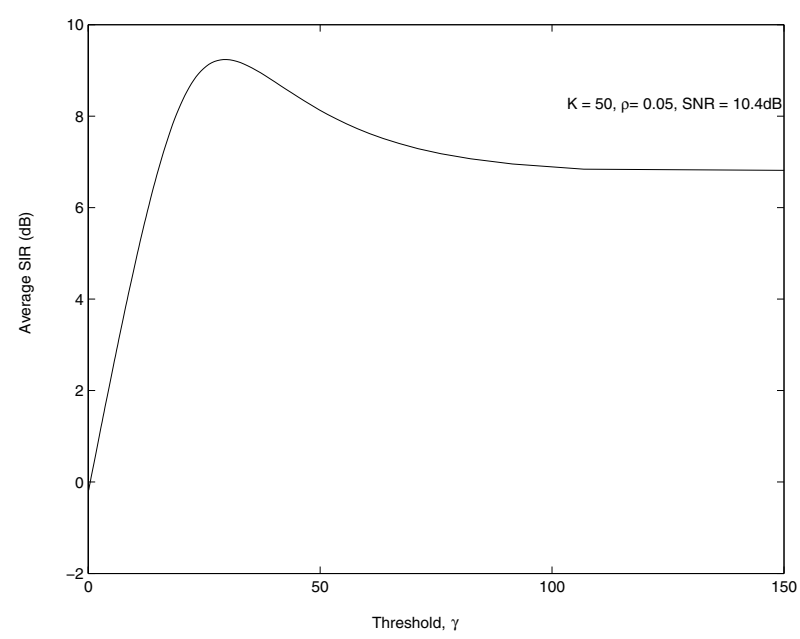

Fig. 1. Average SIR at the canceller output as a function of the threshold $\gamma$. $K=50, \rho=0.05, \mathrm{SNR}=10.4 \mathrm{~dB}$.

$\rho \sum_{\substack{j=1 \\ j \in S}}^{K} y_{j}$, where $S$ denotes the set of the $n_{a}$ users cancelled. The interference variance $\tilde{\sigma}_{I_{2}}^{2}$ of the cancelled output is approximately $\tilde{\sigma}_{I_{2}}^{2}=A^{2} \rho^{4}\left(n_{a}-1\right)^{2} n_{a}+A^{2}(\rho-$ $\left.\rho^{2} n_{a}\right)^{2}\left(K-1-n_{a}\right)$, and the noise variance $\tilde{\sigma}_{N_{2}}^{2}$ is given by $\tilde{\sigma}_{N_{2}}^{2}=\sigma^{2}-\rho^{2} n_{a} \sigma^{2}+\rho^{3} \sigma^{2} n_{a}\left(n_{a}-1\right)$. An approximate expression for the average SIR at the canceller output can then be written as

$$
\overline{S I R}=\frac{A^{2}\left(1-n_{a} \rho^{2}\right)^{2}}{\tilde{\sigma}_{I_{2}}^{2}+\tilde{\sigma}_{N_{2}}^{2}} .
$$

The average SIR as a function of the threshold $\gamma$ for $K=$ 50, $\rho=0.05$, and SNR $=\frac{A^{2}}{\sigma^{2}}=10.4 \mathrm{~dB}$ is shown in Fig. 1. Differentiating (19) w.r.to $n_{a}$ and equating to zero, the optimum value of $n_{a}, n_{a}^{o p t}$, can be obtained as

$$
n_{a}^{o p t}=\frac{U_{1}(K, \rho, \sigma)}{U_{2}(K, \rho, \sigma)},
$$

where $U_{1}(K, \rho, \sigma)=A^{2}-\sigma^{2}(1-\rho)+2 \rho A^{2}(K-1)-$ $\rho^{2} A^{2}(2 K-1)$, and $U_{2}(K, \rho, \sigma)=\rho^{4} A^{2}-2 \rho^{3} A^{2}(K-1)+$ $\rho^{2} A^{2}(2 K-7)+4 \rho A^{2}-\sigma^{2}\left(\rho^{3}+\rho^{2}-2 \rho\right)$. Substituting the value of $n_{a}^{\text {opt }}$ in (18), the optimum value of $\gamma$ can be found for the given channel conditions.

It is noted that both the MF detector as well as the conventional LPIC can be viewed as special cases of the proposed scheme corresponding to $\gamma=\infty$ (none of the users get cancelled) and $\gamma=0$ (all users get cancelled), respectively. Also, by substituting $n_{a}=K-1$, (19) reduces to the SIR expression in (10) corresponding to the conventional LPIC, and by substituting $n_{a}=0$ it reduces to the SIR expression corresponding to the MF detector in [1].

Next consider the non-equi-correlated case. Let the $k$ th user be the desired user. We are interested in writing an optimizing function for the interfering user $j$ to obtain the threshold value $\gamma_{j}$ for the user $j$. For this, we take the mean square error (MSE) expression for the $j$ th user in a conventional LPIC scheme [5] and modify it as follows. Let $X_{j}$ denote the event $\frac{A_{j}^{2}\left|h_{j}\right|^{2}}{\sigma^{2}}<\gamma_{j}$ (i.e., user $j$ is not cancelled with 
probability $\left.p\left(X_{j}\right)\right)$, and $\overline{X_{j}}$ denote the event $\frac{A_{j}^{2}\left|h_{j}\right|^{2}}{\sigma^{2}} \geq \gamma_{j}$ (i.e., user $j$ is cancelled with probability $P\left(\overline{X_{j}}\right)$ ). Note that $P\left(\overline{X_{j}}\right)=e^{-\frac{\gamma_{j} \sigma^{2}}{2 A_{j}^{2}}}$. Now, consider the function $Z_{j}$ given by

$$
\begin{aligned}
Z_{j}= & P\left(\overline{X_{j}}\right) E\left[\left|\rho_{j k} \sum_{\substack{i=1 \\
i \neq k, j}}^{K} A_{i} h_{i} b_{i} \rho_{i j}+\rho_{j k} n_{j}\right|^{2} \mid \overline{X_{j}}\right] \\
& +P\left(X_{j}\right) E\left[A_{j}^{2}\left|h_{j}\right|^{2} \rho_{j k}^{2} \mid X_{j}\right],
\end{aligned}
$$

where the first term accounts for the MSE of the $j$ th user's MAI estimator when user $j$ is cancelled, and the second term accounts for the interference introduced by the $j$ th user when he is not cancelled. The first conditional expectation in the above can be found by averaging $\left|h_{j}\right|^{2}$ using the conditional distribution $f\left(\left|h_{j}\right|^{2} \mid X_{j}\right)$. The second expectation can be found by noting that the terms involved are independent of the event $X_{j}$. The $Z_{j}$ can then be written as

$$
\begin{aligned}
Z_{j}= & P\left(\overline{X_{j}}\right)\left(\rho_{j k}^{2} \sum_{\substack{i=1 \\
i \neq k, j}}^{K} 2 A_{i}^{2} \rho_{i j}^{2}+2 \rho_{j k}^{2} \sigma^{2}\right) \\
& +A_{j}^{2} \rho_{j k}^{2}\left(2-2 P\left(\overline{X_{j}}\right)-\gamma_{j} \sigma^{2} P\left(\overline{X_{j}}\right)\right)
\end{aligned}
$$

which can be optimized to obtain the threshold $\gamma_{j}$. Alternately, we can optimize the following function $\tilde{Z}_{j}$ which includes additional cross-terms that make the sum of $Z_{j}$ 's when $\gamma_{j}$ is zero close to the conventional LPIC interference variance

$$
\begin{aligned}
\tilde{Z}_{j}= & P\left(\bar{X}_{j}\right)\left(\rho_{j k}^{2} \sum_{\substack{i=1 \\
i \neq k, j}}^{K} 2 A_{i}^{2} \rho_{i j}^{2}+2 \rho_{j k}^{2} \sigma^{2}\right) \\
& +2 \rho_{j k} P\left(\overline{X_{j}}\right) \sum_{\substack{i=1 \\
i \neq j, k}}^{K} \sum_{\substack{l=1 \\
l \neq i, k, j}}^{K} A_{i}^{2} \rho_{l k} \rho_{i j} \rho_{i l} \\
& +A_{j}^{2} \rho_{j k}^{2}\left(2-2 P\left(\bar{X}_{j}\right)-\gamma_{j} \sigma^{2} P\left(\bar{X}_{j}\right)\right) .
\end{aligned}
$$

For the case of diversity channels, the above optimization can be carried out on the individual fading paths.

\section{Performance Results}

In Fig. 2, we plot the bit error rate as a function of the number of users, $K$, for $a$ ) the MF detector, $b$ ) the conventional LPIC, and $c$ ) the proposed threshold-based LPIC, for the case of equi-correlated users with $\rho=0.05$ at an SNR of 10.4 $\mathrm{dB}$ on flat Rayleigh fading channels $(L=1)$. While the performance curves for the conventional LPIC and the MF detector are obtained from analytical expressions (Eqn. (11) for the conventional LPIC and Eqn. (3.135) in [1] for the MF detector), the performance curve for the threshold-based LPIC is obtained through simulations. We have also verified the analytical plots for the conventional LPIC and the MF detector with simulation results and found the analysis and simulation results to agree very well (since the BER expressions are exact and no approximation is involved). The $\gamma$ that

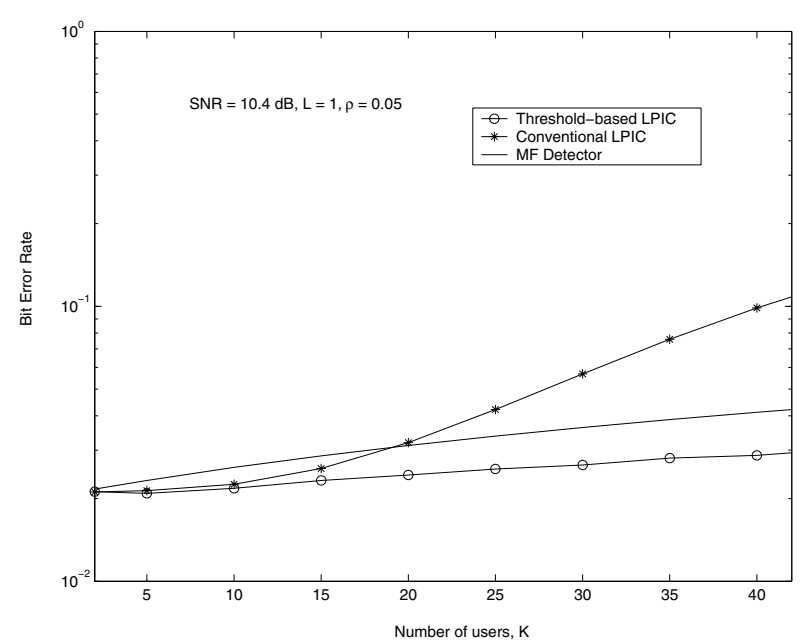

Fig. 2. Comparison of the BER performance of the threshold-based LPIC scheme with that of the MF detector and the conventional LPIC on flat Rayleigh fading $(L=1)$. Equi-correlated users with $\rho=0.05$. SNR $=$ $10.4 \mathrm{~dB}$.

maximizes the output SIR in (19) is chosen as the optimum threshold for the proposed scheme.

As mentioned earlier, from Fig. 1 we observe that the conventional LPIC performs worse than the MF detector when $K$ is large (i.e., inaccurate MAI estimates due to poor SIR condition), whereas it performs better than the MF detector when $K$ is small. It is also observed that the proposed thresholdbased LPIC performs better than both the MF detector as well as the conventional LPIC. This is because users with possibly very inaccurate MAI estimates (such users identified by their instantaneous SNRs falling below the optimum threshold) are not cancelled in the proposed scheme. As pointed out earlier, the MF detector and the conventional LPIC can be viewed as special cases corresponding to $\gamma=\infty$ and $\gamma=0$, respectively, whereas the proposed scheme uses the optimum $\gamma$ which maximizes the output SIR and hence performs better than both the MF and the conventional LPIC. Figure 3 shows a similar performance comparison on diversity channels with two independent fading paths $(L=2)$. Figure 4 shows the BER versus SNR performance comparison of the three schemes for 8 equi-correlated users with $\rho=0.125$. We observe that the proposed LPIC scheme performs better than the MF detector and the conventional LPIC.

Figures 5 and 6 illustrate performance of the proposed scheme for the non-equi-correlated users case on flat fading $(L=1)$ and diversity channels $(L=2)$, respectively, at an SNR of $10.4 \mathrm{~dB}$. Random spreading sequences of processing gain of 32 are used. The optimum threshold for each user is obtained by optimizing (23). In the simulations, a near-far scenario is considered with users 2,4 , and 5 transmitting with 10 times more amplitude than the desired user 1 (i.e., $A_{2} / A_{1}=$ $\left.A_{4} / A_{1}=A_{5} / A_{1}=10\right)$ and the remaining users transmit with the same amplitude as the desired user. It is observed that in the presence of near-far effect, the MF detector performs worse than the conventional LPIC in the range of number of users considered. However, the proposed thresholdbased LPIC scheme clearly outperforms both the MF detector as well as the conventional LPIC. 


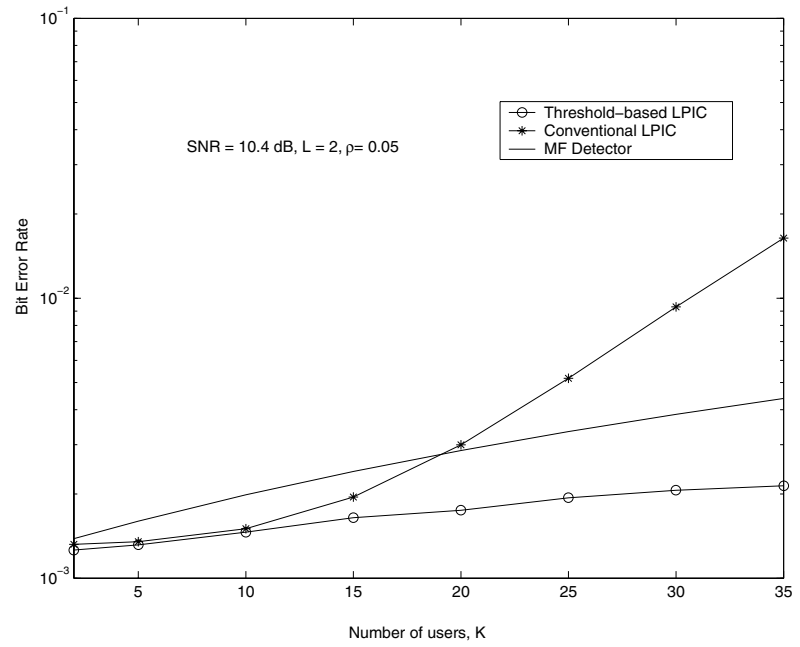

Fig. 3. Comparison of the BER performance of the threshold-based LPIC scheme with that of the MF detector and conventional LPIC on diversity channels $(L=2)$. Equi-correlated users with $\rho=0.05$. SNR $=10.4 \mathrm{~dB}$.

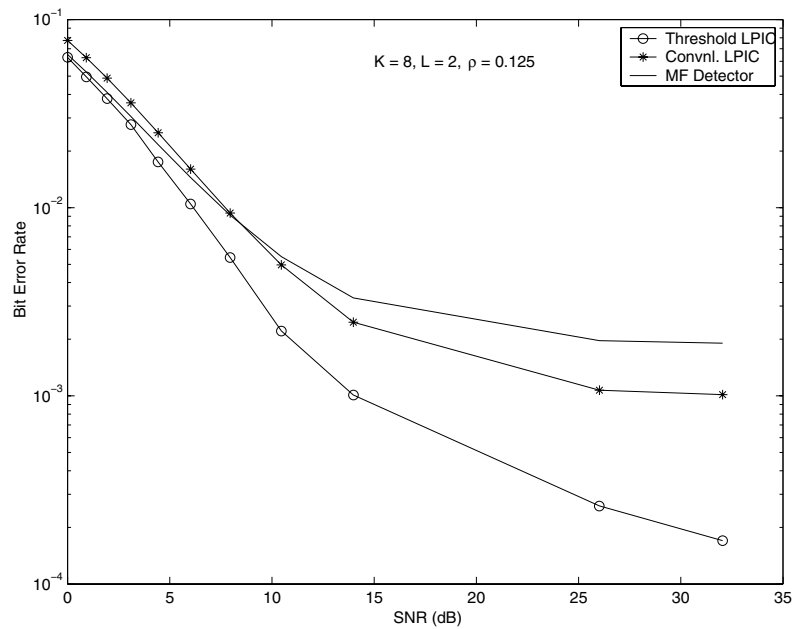

Fig. 4. BER versus SNR performance of the threshold-based LPIC, the conventional LPIC, and the MF detector on diversity channels $(L=2)$. 8 equi-correlated users with $\rho=0.125$.

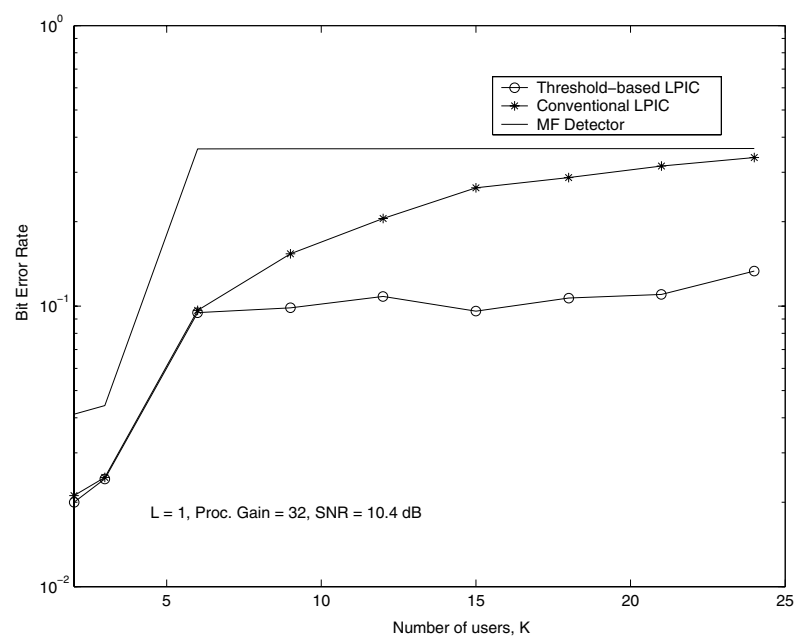

Fig. 5. Comparison of the BER performance of the threshold-based LPIC scheme with that of the MF detector and conventional LPIC on flat fading channels $(L=1)$. Non-equi-correlated users. SNR $=10.4 \mathrm{~dB}$. Processing gain $=32$. Random spreading sequences. Users 2, 4, 5 transmit with 10 times more amplitude than the other users.

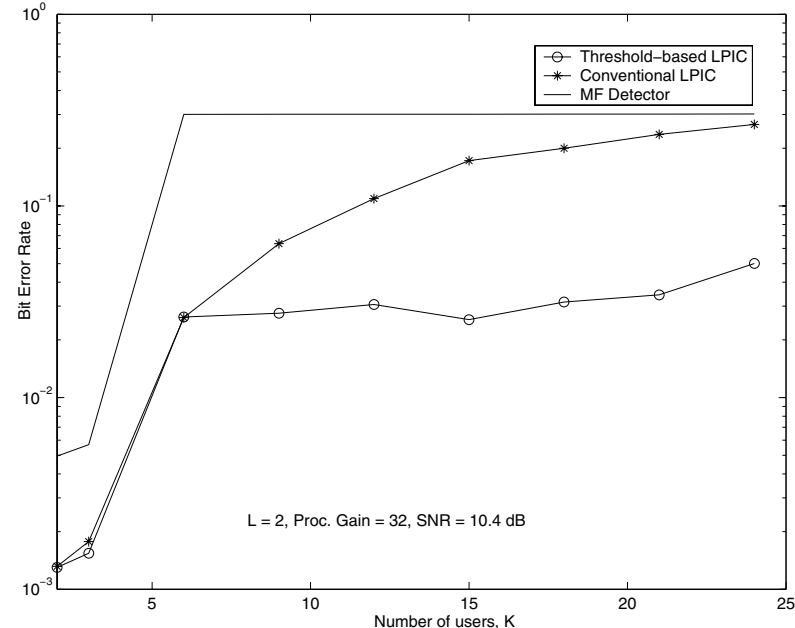

Fig. 6. Comparison of the BER performance of the threshold-based LPIC scheme with that of the MF detector and conventional LPIC on diversity channels $(L=2)$. Non-equi-correlated users. SNR $=10.4 \mathrm{~dB}$. Processing gain $=32$. Random spreading sequences. Users 2, 4, 5 transmit with 10 times more amplitude than the other users.

\section{CONCLUSION}

The contributions in this paper were twofold; first, we obtained closed-form expressions for the BER of conventional LPIC, where the interference from all other users are estimated and cancelled on Rayleigh fading and diversity channels, and second, we also proposed an improved LPIC scheme which cancels the interference from only those users whose instantaneous SNRs exceed a certain threshold on Rayleigh fading channels. The optimum threshold was obtained by optimizing an approximate SIR expression at the canceller output. We showed that the proposed threshold-based LPIC scheme performs better than the matched filter detector as well as the conventional LPIC scheme.

\section{REFERENCES}

[1] S. Verdu, Multiuser Detection, Cambridge University Press, 1998.

[2] G. B. Ginnakis, Y. Hua, P. Stoica, and L. Tong, Signal Processing Advances in Wireless \& Mobile Communications Vol .2: Trends in Singleand Multi-user Systems, Prentice Hall, NJ 2000.

[3] M. Varanasi and B. Aazhang, "Multistage detection in asynchronous code-division multiple-access," IEEE Trans. Commun., vol. 38, pp. 509-519, April 1990.

[4] R. Chandrasekaran and J. J. Shynk, "Analysis of parallel interference canceller for DS-CDMA signals," Proc. 37th Allerton Conf. on Communication, Control and Computing, Urbana, IL, September 1999.

[5] D. R. Brown, M. Motani, V. Veeravalli, H. V. Poor, and C. R. Johnson, Jr., "On the performance of linear parallel interference cancellation," IEEE Trans. on Information Theory, vol. 47, no. 5, pp. 1957-1970, July 2001.

[6] D. Guo, L. K. Rasmussen, and T. J. Lim, "Linear parallel interference cancellation in long-code CDMA multiuser detection," IEEE Jl. Sel. Areas in Commun., vol. 17, no. 12, pp. 2074-2081, December 1999.

[7] D. Divsalar, M. K. Simon, and D. Raphaeli, "Improved parallel interference cancellation for CDMA," IEEE Trans. Commun., vol. 46, no. 2, pp. 258-268, February 1998.

[8] W. Tantiphaiboontana and S. L. Miller, "Performance analysis of partial parallel interference cancellation in synchronous DS-CDMA systems over frequency selective fading channels," Proc. IEEE ICC'2003, pp. 2557-2561, May 2003. 